\title{
PREGNANCY WITH HEART DISEASE - FETOMATERNAL OUTCOME
}

Mainak Sen ${ }^{1}$, Parnamita Bhattacharyya ${ }^{2}$, Nilanjana Chowdhury ${ }^{3}$

\section{HOW TO CITE THIS ARTICLE:}

Mainak Sen, Parnamita Bhattacharyya, Nilanjana Chowdhury. "Pregnancy with Heart Disease - Fetomaternal Outcome". Journal of Evolution of Medical and Dental Sciences 2014; Vol. 3, Issue 05, February 03; Page: 11781183, DOI: $10.14260 /$ jemds/2014/1964

ABSTRACT: OBJECTIVES: To evaluate the maternal and fetal outcome of pregnancies complicated by cardiac disease in a developing country. METHODS: A prospective analysis was carried out of 40 pregnancies in women with cardiac disease who delivered at 28 weeks of gestation and beyond from June 2009 to May 2010 at a tertiary care center in the eastern part of India. RESULTS: Rheumatic heart disease $(n=28,70 \%)$ with isolated mitral stenosis $(n=21)$ was the predominant cardiac problem. Septal defects were the most common form of congenital heart disease $(n=10)$. In 28 $(13.52 \%)$ women, the diagnosis of cardiac disease was made during pregnancy. Patients in NYHA class I/II ( $\mathrm{n}=29,72.5 \%)$ had fewer maternal complications and their babies had a higher birth weight than those in NYHA class III/IV ( $n=11,27.5 \%)$. Cardiac complications were noted in 27 (67.5\%) patients. Commonest complication developing during pregnancy, labor and puerperium was congestive cardiac failure $(n=14,35 \%)$. Maternal mortality was noted in 3 patients $(7.5 \%), 2$ of which were due to cardiac failure and pulmonary edema. Six patients (15\%) delivered preterm and thirteen patients (32.5\%) had low birth weight babies. There were three neonatal deaths and one stillborn. CONCLUSIONS: Rheumatic heart disease was the predominant type. Patients in NYHA class I/II had a better maternal and fetal outcome than those in NYHA class III/IV. Surgically treated women tolerate pregnancy well. Vaginal delivery was safer and caesarean section should be reserved only for obstetric indications. Maternal and perinatal outcome can be improved by team approach at tertiary care center.

KEYWORDS: Pregnancy, heart disease, fetomaternal outcome.

INTRODUCTION: Pregnancy in woman with heart disease increases the risk of maternal and fetal complications. About $1 \%$ of pregnant women have concomitant cardiac disease 1 . Acute rheumatic fever and rheumatic valvular disease remain prevalent in many parts of the world, and are the most common cause of heart disease in pregnancy ${ }^{2}$. This population of pregnant women with valvular heart disease represents a unique patient group with increased risk for adverse outcomes. The significant hemodynamic changes that occur during pregnancy can mimic symptoms of congestive heart failure. Furthermore, many patients with valvular heart disease are first recognized during pregnancy. Pre-pregnancy intervention is of utmost importance in high-risk women who present for evaluation before a planned pregnancy. Moreover better medical management and newer surgical techniques has enabled more women with congenital heart disease to reach childbearing age. Objective of the present study was to find out pregnancy complications, mode of delivery and fetomaternal outcome in pregnancy with valvular and congenital heart diseases.

METHODS: This was a prospective analytical study conducted in the department of Obstetrics and Gynecology, NRS Medical College and Hospital from June 2009 to May 2010. Forty pregnant women with various types of cardiac lesions were included in the study. Thirty two, out of these forty 
patients were booked cases and remaining eight cases were admitted directly in the hospital with labor pain or heart failure.

Functional grading of all cases was done in antenatal and postpartum period according to the NYHA classification. Patients were referred to cardiologist after their first visit and thereafter managed by joint supervision of obstetrician and cardiologist throughout their pregnancy. History of rheumatic fever, cardiac operations, and previous pregnancy complications was detailed. Apart from routine antenatal investigations, ECG and echocardiography were done and repeated when necessary.

Patients with NYHA class I disease were admitted two weeks prior to their expected date of delivery, patients with NYHA class II disease were admitted between 32-34 weeks of gestation or earlier in presence of complication, and patients with class III and IV disease were admitted as soon as they were diagnosed.

Delivery was planned accordingly and patients were kept in hospital for at least seven days postpartum or more if required. Fetal outcome was noted in terms of birth weight, maturity, Apgar score, congenital anomaly.

RESULTS: Most (70\%) of the patients in this study were in the age group of 20-29 years. Twelve patients had congenital heart disease, of which 6 patients had ASD, 4 patients had VSD, 1 patient had coarctation of aorta with bicuspid aortic valve and 1 patient had corrected Fallot's tetralogy. Rest 28 patients $(70 \%)$ had valvular heart disease, all of rheumatic origin. Isolated mitral valve involvement was found in 19 cases, and 9 cases had combined involvement of mitral and aortic valves. Prior history of cardiac surgery was present in 8 patients. It included 3 cases of congenital heart disease, one for correction of ASD, and one for VSD and another for correction of Fallot's tetralogy. Five cases of rheumatic heart disease were operated of which 2 had closed mitral valvotomy and were almost symptomless. Both maternal and fetal outcome were better in operated cases.

Majority of the patients (18) belonged to NYHA class II. 11 patients had NYHA class I, 7 patients had NYHA class III and 4 patients had NYHA class IV disease. Most common symptom on admission was dyspnea (20 cases). Other symptoms were cough (10cases), pedal edema (8cases), cyanosis ( 3 cases), syncopal attack ( 2 cases), and palpitation (10 cases).

In the present series, 27 out of 40 patients had associated cardiac complications either preexistent or developed during pregnancy, labor or puerperium. Eighteen patients had pulmonary arterial hypertension of varying severity. Congestive cardiac failure developed in 14 cases, of which 6 patients suffered pulmonary edema. Atrial fibrillation and supraventricular tachycardia were seen in 10 and 6 cases respectively. A total of 6 patients had systemic hypertension. None of the patients developed bacterial endocarditis. (Table 1 )

Other complications like anemia and respiratory tract infection was present in $37.5 \%$ and $25 \%$ cases respectively. Preeclampsia developed in $7.5 \%$ patients.

Most of the patients (55\%) were treated with adequate rest, digoxin therapy, long acting benzathine penicillin and furosemide diuretics and endocarditis prophylaxis during labor. No patients were treated with anticoagulants or required any cardiac interventions.

Twenty seven patients (67.5\%) delivered vaginally. Outlet forceps were given in 24 patients to cut short the second stage of labor and low forceps was given in one case with fetal distress. Rest of the two patients had spontaneous vaginal delivery. Lower segment caesarean sections were done in 
12 cases, (7 elective and 5 emergency LSCS). One post-mortem caesarean section was also performed. Indication of elective LSCS was gross IUGR in a patient with ASD, one patient of secondary infertility with Cephalopelvic disproportion, to avoid postdatism in a patient with rhesus negative pregnancy, coarctation of aorta in one patient and severe pulmonary artery hypertension in three patients. Indication of emergency LSCS were fetal distress in two patients, non-progress of labor in two patients and post caesarean pregnancy with scar tenderness in one patient. In all cases epidural anesthesia was used except in two cases of fetal distress where general anesthesia was performed. None of the patients undergoing LSCS developed any complication.

In the present study three maternal deaths occurred out of 40 patients (7.5\%). One death occurred antenatally in an unbooked patient with multivalvular heart disease and atrial fibrillation. Two maternal deaths occurred postnatally, one 3 hours after delivery and another on second day after delivery due to heart failure precipitated by severe anemia in mutivalvular heart disease.

Overall perinatal outcome was good. 100\%of NYHA class I and class II patients delivered between $37-40$ weeks compared to $57.1 \%$ of class III and $25 \%$ of class IV patients. Six patients (15\%) delivered preterm and thirteen patients (32.5\%) had low birth weight babies. There were three neonatal deaths and one stillborn. One neonate died due to prematurity, one due to septicemia and the other one due to meconium aspiration. The stillborn baby was a diagnosed case of intrauterine fetal death in a booked mother with mitral stenosis. One baby, whose mother had ASD, was diagnosed to have membranous type of VSD.

DISCUSSION: Heart disease in pregnancy is an uncommon problem in the developed world but reaches a high prevalence in developing countries. With the advent of more and more modern techniques and advancement in knowledge, the hemodynamic circulation in pregnancy is now better understood and managed. The availability of echocardiography provides information about disease etiology, accurate and noninvasive assessment of severity and means of monitoring progression. Contraindications for pregnancies still remain severe pulmonary artery hypertension, Eisenmengersyndrome and severe non corrected cyanotic disease as well ${ }^{1}$. In developed countries, congenital heart diseases are the commonest cause of cardiopathy in pregnant women ${ }^{3}$. However rheumatic heart disease still remains the most common cardiopathy found in pregnant woman in our country.

In our prospective analytical study conducted over a period of one year, 40 cases of different types of cardiac lesions with pregnancy were included. In $70 \%$ of the patients, heart disease was of rheumatic origin and rest $30 \%$ of the patients presented with congenital heart disease. Mitral stenosis was the commonest valvulopathy $(n=21)$. This was consistent with a previous study conducted by Doshi $\mathrm{Hu}$ et al where rheumatic cardiac disease (68.62\%) with mitral valve involvement $(88.57 \%)$ was the commonest cardiac disease in pregnancy ${ }^{4}$. In our study, five cases of rheumatic heart disease were previously operated but none of the patients had history of valve replacement and therefore no patients required anticoagulant therapy.

Among the congenital heart diseases ASD and VSD constituted 83.3\%cases (10/12). This incidence was greater than that found in a previous study by Meng Y et al where ASD and VSD constituted $58 \%$ of congenital heart disease patients ${ }^{5}$. This difference may be due overall small population of congenital heart disease patients in the present study.

Cardiac complications were present in $67.5 \%$ patients $(27 / 40)$. These included cases of pulmonary hypertension $(n=18)$, congestive cardiac failure $(n=14)$, pulmonary edema $(n=6)$, cardiac 
arrhythmias like atrial fibrillation $(\mathrm{n}=10)$ and supraventricular tachycardia $(\mathrm{n}=6)$. Although pulmonary hypertension was more prevalent, commonest complication developed during pregnancy, labor and puerperium was heart failure (35\%). This was similar to the findings of Doshi HU et al ${ }^{4}$ and heart failure was more common in NYHA grade III and IV groups.

Vaginal delivery occurred in $67.5 \%$ patients. Rest of the patients underwent LUCS. Indication for performing elective and emergency LUCS were mainly obstetrical. The only cardiac indications were coarctation of aorta $(n=1)$, and severe pulmonary artery hypertension $(n=3)$. Preterm delivery occurred in $15 \%$ patients and all of them belonged to NYHA class III and IV group ( $\mathrm{p}=.03)$. Average birth weight in NYHA class I and II was $2.8 \mathrm{~kg}$ whereas average birth weight in NYHA class III and IV was $2.2 \mathrm{~kg}(\mathrm{p}=.04)$. In a previous study of valvular heart disease by Leśniak-Sobelga A et al $80 \%$ of all deliveries occurred vaginally and incidence of preterm labor was $4 \%{ }^{6}$. In other studies conducted by Lin JH et al CS rate was 79\% and preterm labor occurred in 35\% cases of heart disease complicated by pulmonary hypertension ${ }^{7}$ and in cases of pregnancy complicated by rheumatic heart disease, the delivery week was 34.6 and the birth weight was $2176 \mathrm{~g}$ averagely in NYHA class IV group and had significant differences from NYHA class I group $(\mathrm{P}<0.05)^{8}$.

In the present study, apart from one case of VSD, no other congenital anomalies were found in the newborns. Maternal mortality occurred in $7.5 \%$ cases. This was consistent with a previous study by Naidoo DP et al where there was high maternal mortality rate of $9.5 \%$ and rheumatic heart disease accounted for most of them.

CONCLUSION: The management of pregnant woman with heart disease requires a multidisciplinary team for optimal maternal and fetal outcome. Acute rheumatic fever and chronic rheumatic valvular disease in their most virulent form are still commonly encountered and impose a huge burden on limited healthcare resources. Mitral stenosis is the most frequently encountered rheumatic valvular lesion. The use of percutaneous mitral balloon valvuloplasty has improved the obstetric outcome of mitral stenosis in symptomatic patients. Severe aortic stenosis is associated with poor prognosis. Regurgitant valvular diseases are often well tolerated with medical therapy. A variety of pregnancyassociated cardiovascular changes often exacerbate the signs and symptoms of valvular lesions. Pregnancy should not be allowed to proceed, if possible, in patients with uncorrected severe valvular lesions, as maternal and fetal morbidity and mortality are high. For those with milder disease, pregnancy is best undertaken after the valvular lesion has been rectified or stabilized. Besides management during the antenatal period, timing and mode of delivery should be decided upon jointly by the obstetrician, cardiologist, and obstetric anesthesiologist.

Pregnant women with rheumatic heart disease of moderate-severe mitral stenosis, severe pulmonary hypertension and atrial fibrillation are at high risk of heart failure. The fetal outcome is not good in cases of NYHA class III and IV

Surgically treated women tolerate pregnancy well. Vaginal delivery is safer and caesarean section should be reserved only for obstetric indications. Maternal and perinatal outcome can be improved by team approach at tertiary care center.

\section{BIBLIOGRAPHY:}

1. Zuber M, Jenni R. Heart valve disease in women in the reproductive age. Ther Umsch. 1998 Dec; 55(12):762-6. 
2. Lim ST. Rheumatic heart diseases in pregnancy. Ann Acad Med Singapore. 2002 May; 31(3):340-8.

3. Fennira S, Rejeb MA, Ellouze Y, Khaldi H. Heart diseases in pregnant women. Tunis 2008 Jun; 86(6):584-90.

4. Doshi HU, Oza HV, Tekani H, Modi K. Cardiac disease in pregnancy--maternal and perinatal outcome. J Indian Med Assoc. 2010 May;108(5):278-80, 282

5. Meng Y, Huang YP, Liu XL. Perinatal outcomes of 45 pregnant women with pulmonary hypertension complicating congenital heart disease. Zhonghua Fu Chan Ke Za Zhi. 2007 Oct; 42(10):662-5.

6. Leśniak-Sobelga A, Tracz W, KostKiewicz M. Clinical and echocardiographic assessment of pregnant women with valvular heart diseases--maternal and fetal outcome. Int J Cardiol. 2004 Mar; 94(1):15-23.

7. Lin JH, Zhao WX, Su Y. Perinatal management and pregnancy outcome in pregnant women with pulmonary hypertension complicating cardiac disease. Zhonghua Fu Chan Ke Za Zhi. 2006 Feb; 41(2):99-102.

8. Lin JH, Ling WW, Liang AJ. Pregnancy outcome in women with rheumatic heart disease. Zhonghua Fu Chan Ke Za Zhi. 2007 May; 42(5):315-9.

\begin{tabular}{|c|c|c|c|c|c|c|}
\hline Total pts. & \multicolumn{4}{|c|}{ Congenital heart ds (30\%) } & \multicolumn{2}{c|}{ Rheumatic heart ds (70\%) } \\
\hline \multirow{2}{*}{40} & ASD & VSD & Coarctation of aorta & Corrected Fallot's & Mitral & Mitral +Aortic \\
\cline { 2 - 7 } & 6 & 4 & 1 & 1 & $19(48 \%)$ & $9(23 \%)$ \\
\hline
\end{tabular}

Table-1

\begin{tabular}{|c|c|c|c|c|}
\hline NYHA & I & II & III & IV \\
\hline Total (40) & 11 & 18 & 7 & 4 \\
\hline \multicolumn{4}{|c|}{ Table-2 } \\
\hline
\end{tabular}

\begin{tabular}{|c|c|c|c|c|c|}
\hline \multicolumn{3}{|c|}{ Vaginal deliveries (68\%) } & \multicolumn{3}{c|}{ LSCS (32\%) } \\
\hline Normal delivery & \multicolumn{2}{|c|}{ Forceps (63\%) } & Emergency & Elective & Post mortem CS \\
\hline \multirow{2}{*}{2} & Outlet & Low & 5 & 7 & 1 \\
\cline { 2 - 3 } & 24 & 1 & 5 & & \multirow{2}{*}{ Table-3 } \\
\hline
\end{tabular}

\begin{tabular}{|c|c|c|c|}
\hline Maternal outcome & Antenatal deaths & Postpartum deaths & No/Minimal complications \\
\hline Total $(40)$ & $1(2.5 \%)$ & $2(5 \%)$ & $37(92.5 \%)$ \\
\hline \multicolumn{4}{|c|}{ Table-4 } \\
\hline
\end{tabular}




\section{ORIGINAL ARTICLE}

\section{AUTHORS:}

1. Mainak Sen

2. Parnamita Bhattacharyya

3. Nilanjana Chowdhury

\section{PARTICULARS OF CONTRIBUTORS:}

1. RMO cum Clinical Tutor, Department of Obstetrics and Gynaecology, Malda Medical College and Hospital.

2. Assistant Professor, Department of Obstetrics and Gynaecology, Calcutta Medical College and Hospital.

3. Professor, Department of Obstetrics and Gynaecology, R.G. Kar Medical College and Hospital.

\section{NAME ADDRESS EMAIL ID OF THE CORRESPONDING AUTHOR:}

Dr. Mainak Sen, Neelachal Abasan Co-Operative Society, 98, Rajdanga Gold Park,

Flat- Purba B4,

Kolkata - 107.

E-mail: mainak_sen1977@yahoo.co.in

Date of Submission: 11/01/2014.

Date of Peer Review: 12/01/2014.

Date of Acceptance: 21/01/2014.

Date of Publishing: 29/01/2014. 